Groups Geom. Dyn. 4 (2010), 309-332

DOI $10.4171 / \mathrm{GGD} / 85$
Groups, Geometry, and Dynamics

(C) European Mathematical Society

\title{
Amenable actions of amalgamated free products
}

\author{
Soyoung Moon*
}

\begin{abstract}
We prove that the amalgamated free product of two free groups of rank two over a common cyclic subgroup admits an amenable, faithful, transitive action on an infinite countable set. We also show that any finite index subgroup admits such an action, which applies for example to surface groups and fundamental groups of surface bundles over $\mathbb{S}^{1}$.
\end{abstract}

Mathematics Subject Classification (2010). 43A07, 20E06, 57M07.

Keywords. Amenable actions, amalgamated free products.

\section{Introduction}

An action of a group $G$ on a set $X$ is amenable if there exists a $G$-invariant mean on $X$, i.e., a map $\mu: 2^{X}=\mathcal{P}(X) \rightarrow[0,1]$ such that $\mu(X)=1, \mu(A \cup B)=\mu(A)+\mu(B)$ for every pair of disjoint subsets $A, B$ of $X$, and $\mu(g A)=\mu(A)$ for all $g \in G$ and for all $A \subseteq X$.

The study of amenability goes back to von Neumann [13] and has spanned over the 20th century in various fields of mathematics, such as geometric group theory, harmonic analysis, graph theory, operator algebra, etc. F. P. Greenleaf asked in [8] whether the presence of a $G$-invariant mean on a set on which $G$ acts faithfully implies that the group $G$ is amenable (i.e., if the action on itself by left multiplication is amenable), and the first counter example was given in [5], where E. K. van Douwen constructed an interesting amenable action of the non-abelian free group.

The above definition is due to Greenleaf [8]. We should mention that Zimmer [17] has also introduced a notion of amenability for a group action that is different from ours; an action by homeomorphisms of a countable discrete group $G$ on a compact Hausdorff space $X$ is (topologically) Zimmer amenable if there exists a sequence of continuous maps $m^{n}: X \rightarrow \operatorname{Proba}(G)$ such that $\lim _{n \rightarrow \infty} \sup _{x \in X}\left\|g m_{x}^{n}-m_{g x}^{n}\right\|_{1}=$ 0 for all $g \in G$ (cf. [14], [10], [2]). With this definition, a group is amenable if and only if the action on an one-point space is Zimmer amenable, while such an action is always Greenleaf amenable. On the other hand, the action of $G$ on itself by left

\footnotetext{
*This work is supported by Swiss NSF grant 20_118014/1.
} 
multiplication is always Zimmer amenable (by taking $m^{n}: G \rightarrow \operatorname{Proba}(G)$ defined by $m_{g}^{n}=\delta_{g}$ ). More generally, the action of $G$ on a homogenous space $G / H$ is Zimmer amenable if and only if the subgroup $H$ is amenable. From now on, we will use the term of an amenable action as mean of Greenleaf amenable action.

For the study of amenable actions of a group $G$, we should require some restrictions on the $G$-action in order to avoid trivial cases. One should assume that the action is faithful, otherwise one would take immediately a free group $\mathbb{F}_{n}, n \geq 2$, and any non-trivial normal subgroup $N \triangleleft \mathbb{F}_{n}$ such that the quotient group $\mathbb{F}_{n} / N$ is amenable (e.g. $N=\mathbb{F}_{n}^{\prime}$ the commutator subgroup), so that the natural action of $\mathbb{F}_{n}$ on $\mathbb{F}_{n} / N$ is amenable but not faithful. In addition, one should require that $G$ acts transitively, otherwise one could take any group $G$ and $X=G \sqcup Y$ where $G$ acts on $Y$ amenably, so that the $G$-action on $X$ is faithful and amenable (since there is a $G$-equivariant map from $Y$ into $X$ ). In this direction, Y. Glasner and N. Monod [7] proposed to study the class $\mathcal{A}$ of all countable groups which admit a faithful, transitive and amenable action. The class $\mathcal{A}$ is closed under direct products and free products, and a group is in $\mathcal{A}$ if it has a co-amenable subgroup which is in $\mathcal{A}$ (Proposition 1.7 in [7]). On the other hand, in general the class is neither closed under passing to subgroups (the case of finite index subgroups is open), nor closed under semidirect products. As an example for semidirect product, one may take the group $\mathrm{SL}_{2}(\mathbb{Z}) \ltimes \mathbb{Z}^{2}$; while $\mathrm{SL}_{2}(\mathbb{Z})$ is in $\mathcal{A}$ since it contains a free group of finite index, the pair $\left(\mathrm{SL}_{2}(\mathbb{Z}) \ltimes \mathbb{Z}^{2}, \mathbb{Z}^{2}\right)$ has the relative property (T) (cf. [3]), so that the group $\mathrm{SL}_{2}(\mathbb{Z}) \ltimes \mathbb{Z}^{2}$ is not in $\mathcal{A}$ (Lemma 4.3 in [7]). Besides, this group is another example which shows that the class $\mathcal{A}$ is not closed under amalgamated free products; one may see the group $\mathrm{SL}_{2}(\mathbb{Z}) \ltimes \mathbb{Z}^{2}$ as the amalgamated free product $G *_{A} H$ of $G=\mathbb{Z} / 4 \mathbb{Z} \ltimes \mathbb{Z}^{2}$ and $H=\mathbb{Z} / 6 \mathbb{Z} \ltimes \mathbb{Z}^{2}$ along $A=\mathbb{Z} / 2 \mathbb{Z} \ltimes \mathbb{Z}^{2}$ and notice that the three groups $G, H$ and $A$ are in $\mathcal{A}$ since they are amenable.

In particular, Y. Glasner and N. Monod showed that the free product of any two countable groups is in $\mathcal{A}$ unless one factor has the fixed point property and the other has the virtual fixed point property ${ }^{1}$; for this, they used an argument of genericity in Baire's sense (Theorem 3.3 in [7]). Let us mention that another construction of amenable action of a non-abelian free group is obtained by R. Grigorchuk and V. Nekrashevych in [9].

The main result of this paper is, motivated by this method of genericity, to give another example of non-amenable group which is in $\mathcal{A}$ (see Theorem 17 and Theorem 19):

Theorem. The amalgams $\mathbb{F}_{2} *_{\mathbb{Z}} \mathbb{F}_{2}$ belong to $\mathcal{A}$, where $\mathbb{Z}$ embeds in each factor as subgroup generated by some common word on the generating sets.

Such amalgams are known as doubles of $\mathbb{F}_{2}$. The key point of the proof is to fix a transitive permutation $\beta$ and to take a generic element $\alpha$ (i.e., an element in the

\footnotetext{
${ }^{1}$ A group $G$ has the fixed point property if any amenable $G$-action has a fixed point, and $G$ has the virtual fixed point property if it has a finite index subgroup having the fixed point property.
} 
intersection of countably many generic sets) in order to construct $\mathbb{F}_{2}=\langle\alpha, \beta\rangle$ in a way that the amalgamated free product of two copies of $\mathbb{F}_{2}$ along a cyclic group has the desired properties. Therefore, the difficulty of the proof resides in the choice of the generic sets because they can be very "nasty" (see Proposition 1).

As we mentioned before, in general it is not known whether the class $\mathcal{A}$ is closed under passing to finite index subgroups or not. But it is true for our case (see Theorem 20):

Theorem. For any finite index subgroup $H$ of $\mathbb{F}_{2} * \mathbb{Z} \mathbb{F}_{2}$ as above, $H$ belongs to $\mathcal{A}$.

A surface group $\Gamma_{g}$ is the fundamental group of a closed oriented surface of genus $g \geq 2$. The group $\Gamma_{2}$ can be viewed as an amalgamated free product of two copies of $\mathbb{F}_{2}$ along the subgroup generated by the commutator, i.e., $\Gamma_{2}=\left\langle a_{1}, b_{1}\right\rangle *\langle c\rangle\left\langle a_{2}, b_{2}\right\rangle$ where $c=\left[a_{1}, b_{1}\right]=\left[a_{2}, b_{2}\right]$. For $g \geq 3, \Gamma_{g}$ injects into $\Gamma_{2}$ as a finite index subgroup. Therefore, by applying our results, we have the following theorem (see Theorem 21):

Theorem. The surface group $\Gamma_{g}$ belongs to $\mathcal{A}$ for all $g \geq 2$.

As a corollary, we obtain that the fundamental group of a 3-manifold which virtually fibers over the circle is in $\mathcal{A}$. Indeed, let $M$ be a 3-manifold which fibers over the circle. Then there is a short exact sequence

$$
0 \rightarrow \Gamma_{g} \rightarrow \pi_{1}(M) \rightarrow \mathbb{Z} \rightarrow 0,
$$

so that the subgroup $\Gamma_{g}$ is co-amenable in $\pi_{1}(M)$. Moreover, if $M$ is a 3-manifold which virtually fibers over the circle, then it contains a finite index subgroup which is in $\mathcal{A}$, so that $\pi_{1}(M)$ is also in $\mathcal{A}$. Some examples of the fundamental group of such manifolds are given in [1], which includes the Bianchi groups $\operatorname{PSL}\left(2, \mathcal{O}_{d}\right)$, where $\mathcal{O}_{d}$ is the ring of integers of the imaginary quadratic field $\mathbb{Q}(\sqrt{-d})$ with $d$ a positive integer.

Acknowledgement. I would like to thank Nicolas Monod for suggesting the question and for helpful discussions, Alain Valette for his constant help and encouragement, and the referee for useful comments on the first version of this paper.

\section{Baire spaces}

For the importance of the idea of generic choice, we briefly discuss Baire spaces in this section.

Definition 2.1. A topological space $X$ is a Baire space if every intersection of countably many dense open subsets is dense in $X$. 
Equivalently, $X$ is a Baire space if every union of countably many closed subsets with empty interior has empty interior.

Definition 2.2. A Polish space is a separable completely metrizable topological space, i.e., it is a space homeomorphic to a complete space that has a countable dense subset.

Observe that any closed subspace of a Polish space is Polish.

Let $X$ be an infinite countable set. Equipped with the discrete topology, $X$ is a complete topological space. Let us denote by $X^{X}$ the set of all self-maps of $X$ and endow it with the topology of pointwise convergence (i.e., $\alpha_{n}$ converges to $\alpha$ if for all finite subset $F$ of $X$, there exists $n_{0}$ such that $\left.\alpha_{n}\right|_{F}=\left.\alpha\right|_{F}$, for all $n \geq n_{0}$ ). This is the product of the topologies of $X$. Hence $X^{X}$ is complete being a product of complete spaces, and it is separable and metrizable since it is a countable product of separable, metrizable spaces. So $X^{X}$ is a Polish space and by Baire's theorem it is a Baire space.

Let us denote by $\operatorname{Sym}(X) \subset X^{X}$ the group of permutations of $X$. Equipped with the induced topology of $X^{X}, \operatorname{Sym}(X)$ is a topological group. Indeed, let $\left\{\alpha_{n}\right\}_{n \geq 1}$ be a sequence converging to $\alpha$ in $\operatorname{Sym}(X)$. Let $F \subset X$ be a finite subset of $X$. There exists $n_{0}$ such that $\left.\alpha_{n}\right|_{F \cup \alpha^{-1} F}=\left.\alpha\right|_{F \cup \alpha^{-1} F}$ for all $n \geq n_{0}$. Then for all $x \in F$, we have $\alpha_{n}\left(\alpha^{-1}(x)\right)=\alpha\left(\alpha^{-1}(x)\right)=x$, so $\alpha_{n}^{-1}(x)=\alpha^{-1}(x)$ for all $n \geq n_{0}$. Therefore $\alpha_{n}^{-1}$ converges to $\alpha^{-1}$, so that the map $\alpha \mapsto \alpha^{-1}$ is continuous. Moreover, let $\left\{\beta_{m}\right\}_{m \geq 1}$ be a sequence converging to $\beta$ in $\operatorname{Sym}(X)$. Let $F \subset X$ be a finite subset of $X$. There exists $n_{1}$ such that $\left.\alpha_{n}\right|_{F \cup \beta F}=\left.\alpha\right|_{F \cup \beta F}$ for all $n \geq n_{1}$. In addition, there exists $n_{2}$ such that $\left.\beta_{m}\right|_{F}=\left.\beta\right|_{F}$ for all $m \geq n_{2}$. Then for all $x \in F, \alpha_{n}\left(\beta_{m}(x)\right)=\alpha_{n}(\beta(x))=\alpha \beta(x)$ for all $m \geq \max \left\{n_{1}, n_{2}\right\}$. Therefore $\alpha_{n} \beta_{m}$ converges to $\alpha \beta$, so that the map $(\alpha, \beta) \mapsto \alpha \beta$ is continuous.

Consequently, the injection $i: \operatorname{Sym}(X) \rightarrow X^{X} \times X^{X}, \alpha \mapsto\left(\alpha, \alpha^{-1}\right)$, is a homeomorphism onto its image which is closed. Thus $\operatorname{Sym}(X)$ is a Polish space, in particular it is a Baire space.

Definition 2.3. A subset $Y \subset \operatorname{Sym}(X)$ is called

- meagre or first category if it is a union of countably many closed subsets with empty interior;

- generic or dense $G_{\delta}$ if its complement $\operatorname{Sym}(X) \backslash Y$ is meagre, i.e., it is an intersection of countably many dense open subsets.

By definition of the topology on $\operatorname{Sym}(X)$, a subset $Y \subset \operatorname{Sym}(X)$ has empty interior if for all $\alpha^{\prime} \in Y$ and for all finite subset $F \subset X$, there exists $\alpha \in \operatorname{Sym}(X) \backslash Y$ such that $\left.\alpha^{\prime}\right|_{F}=\left.\alpha\right|_{F}$. 


\section{Construction of $\mathbb{F}_{2}$}

Let $X$ be an infinite countable set. Let $\beta$ be a simply transitive permutation of $X$. Let $c=c(\alpha, \beta)$ be a weakly cyclically reduced word (i.e., if $c=g_{m} \ldots g_{1}$, then $\left.g_{m} \neq g_{1}^{-1}\right)$ on the alphabet $\left\{\alpha^{ \pm 1}, \beta^{ \pm 1}\right\}$ such that $c \notin\langle\beta\rangle$.

Proposition 1. The set

$$
\begin{aligned}
& \mathcal{U}_{1}=\{\alpha \in \operatorname{Sym}(X) \mid \text { for all } w \in\langle\alpha, \beta\rangle \backslash\langle c\rangle \text { there exist infinitely many } \\
& \qquad x \in X \text { such that } c x=x, c w x=w x \text { and } w x \neq x\}
\end{aligned}
$$

is generic in $\operatorname{Sym}(X)$.

Proposition 2. The set

$$
u_{2}=\left\{\alpha \in \operatorname{Sym}(X) \mid \text { for all } k \in \mathbb{Z} \backslash\{0\} \text { there exists } x \in X \text { such that } c^{k} x \neq x\right\}
$$

is generic in $\operatorname{Sym}(X)$.

Note that $\mathcal{U}_{2}$ is the set of $\alpha$ 's such that $c$ has infinite order.

Definition 3.1. Let $c=c(\alpha, \beta)$ be a weakly cyclically reduced word. Let $S(\alpha)$ be the sum of exponents of $\alpha$, and $S(\beta)$ be the sum of exponents of $\beta$. We say that $c$ is special if $c$ is one of the following types:

(1) $S(\alpha)=S(\beta)=0$;

(2) $S(\alpha)$ divides $S(\beta)$.

Let $\left\{A_{n}\right\}_{n=1}^{\infty}$ be a pairwise disjoint Følner sequence for $\beta$, that is

$$
\lim _{n \rightarrow \infty} \frac{\left|A_{n} \Delta \beta \cdot A_{n}\right|}{\left|A_{n}\right|}=0 .
$$

Proposition 3. Let c be a special word. The set

$$
\begin{gathered}
\mathcal{U}_{3}=\left\{\alpha \in \operatorname{Sym}(X) \mid \text { there exists a subsequence }\left\{A_{n_{k}}\right\}_{k=1}^{\infty} \text { of }\left\{A_{n}\right\}_{n=1}^{\infty}\right. \\
\text { such that } A_{n_{k}} \subset \operatorname{Fix}(c) \text { for all } k \geq 1 \\
\text { and } \left.\left\{A_{n_{k}}\right\}_{k=1}^{\infty} \text { is a Folner sequence for } \alpha\right\}
\end{gathered}
$$

is generic in $\operatorname{Sym}(X)$.

Proposition 4. The set

$$
\begin{array}{r}
U_{4}=\{\alpha \in \operatorname{Sym}(X) \mid \text { the } H \text {-action on } X \text { is transitive for } \\
\text { all finite index subgroups } H \text { of }\langle\alpha, \beta\rangle\}
\end{array}
$$

is generic in $\operatorname{Sym}(X)$. 
From the previous four propositions, one deduces immediately:

Corollary 5. Let c be a special word on $\left\{\alpha^{ \pm 1}, \beta^{ \pm 1}\right\}$. Let $\alpha \in \mathcal{U}_{1} \cap \mathcal{U}_{2} \cap \mathcal{U}_{3} \cap \mathcal{U}_{4}$. Then $\langle\alpha, \beta\rangle \simeq \mathbb{F}_{2}$ and the following holds:

(1) The action of $\mathbb{F}_{2}$ on $X$ is transitive and faithful.

(2) For all $w \in\langle\alpha, \beta\rangle \backslash\langle c\rangle$, there exist infinitely many $x \in X$ such that $c x=x$, $c w x=w x$ and $w x \neq x$. In particular, there are infinitely many fixed points of $c$ in $X$.

(3) There exists a pairwise disjoint Folner sequence for $\langle\alpha, \beta\rangle$ which is fixed by $c$.

(4) For all finite index subgroup $H$ of $\langle\alpha, \beta\rangle$, the $H$-action on $X$ is transitive.

3.1. Proofs of Propositions 1 and 2. Propositions 1 and 2 are sufficient conditions for faithfulness of $\mathbb{F}_{2}$-action with some additional "unnatural looking" properties that will be needed for construction of $\mathbb{F}_{2} * \mathbb{Z} \mathbb{F}_{2}$ in Section 4. As we resort to the graph theory for these proofs, we begin by fixing the notations on graphs that will be used in the section. The fundamental notions are based on [15].

3.1.1. Graph extension. A graph $G$ consists of the set of vertices $V(G)$ and the set of edges $E(G)$, and two maps $E(G) \rightarrow E(G), e \mapsto \bar{e}$, such that $\overline{\bar{e}}=e$ and $\bar{e} \neq e$, and $E(G) \rightarrow V(G) \times V(G), e \mapsto(i(e), t(e))$, such that $i(e)=t(\bar{e})$. An element $e \in E(G)$ is a directed edge of $G$ and $\bar{e}$ is the inverse edge of $e$. For all $e \in E(G)$, $i(e)$ is the initial vertex of $e$ and $t(e)$ is the terminal vertex of $e$.

Let $S$ be a set. A labeling of a graph $G=(V(G), E(G))$ on the set $S^{ \pm 1}=S \cup S^{-1}$ is a map

$$
l: E(G) \rightarrow S^{ \pm 1}, \quad e \mapsto l(e),
$$

such that $l(\bar{e})=l(e)^{-1}$. A labeled graph $G=(V(G), E(G), S, l)$ is a graph with a labeling $l$ on the set $S^{ \pm 1}$. A labeled graph is well labeled if for any edges $e$, $e^{\prime} \in E(G),\left[i(e)=i\left(e^{\prime}\right)\right.$ and $\left.l(e)=l\left(e^{\prime}\right)\right]$ implies that $e=e^{\prime}$. If a group $\Gamma=\langle S\rangle$ acts on $X$, a labeled graph with set of vertices $X$ and set of edges $S^{ \pm 1}$ is well labeled if and only if it is a Schreier graph.

A word $w=w_{m} \ldots w_{1}$ on $\left\{\alpha^{ \pm 1}, \beta^{ \pm 1}\right\}$ is called reduced if $w_{k+1} \neq w_{k}^{-1}$ for all $1 \leq k \leq m-1$. A word $w=w_{m} \ldots w_{1}$ on $\left\{\alpha^{ \pm 1}, \beta^{ \pm 1}\right\}$ is called weakly cyclically reduced if $w$ is reduced and $w_{m} \neq w_{1}^{-1}$; this definition allows that $w_{m}$ and $w_{1}$ to be equal. We denote by $|w|$ the word length of $w$. Given a reduced word, we shall define two finite graphs labeled on $\left\{\alpha^{ \pm 1}, \beta^{ \pm 1}\right\}$ as follows:

Definition 3.2. Let $w=w_{m} \ldots w_{1}$ be a reduced word on $\left\{\alpha^{ \pm 1}, \beta^{ \pm 1}\right\}$. The path of $w$ is a finite labeled graph $P\left(w, v_{0}\right)$ consisting of $|w|+1$ vertices and $|w|$ directed edges $\left\{e_{1}, \ldots, e_{m}\right\}$ such that

- $i\left(e_{j+1}\right)=t\left(e_{j}\right)$ for all $1 \leq j \leq m-1$;

- $v_{0}=i\left(e_{1}\right) \neq t\left(e_{m}\right)$; 
- $l\left(e_{j}\right)=w_{j}$ for all $1 \leq j \leq m$.

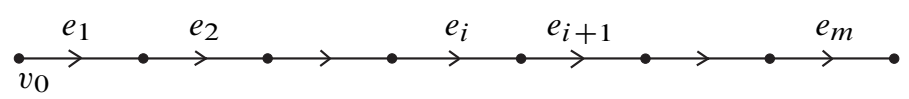

Figure 1. The path of $w$.

Definition 3.3. Let $w=w_{m} \ldots w_{1}$ be a reduced word on $\left\{\alpha^{ \pm 1}, \beta^{ \pm 1}\right\}$. The cycle of $w$ is a finite labeled graph $C\left(w, v_{0}\right)$ consisting of $|w|$ vertices and $|w|$ directed edges $\left\{e_{1}, \ldots, e_{m}\right\}$ such that

- $i\left(e_{j+1}\right)=t\left(e_{j}\right)$ for all $1 \leq j \leq m-1$;

- $v_{0}=i\left(e_{1}\right)=t\left(e_{m}\right)$;

- $l\left(e_{j}\right)=w_{j}$ for all $1 \leq j \leq m$.

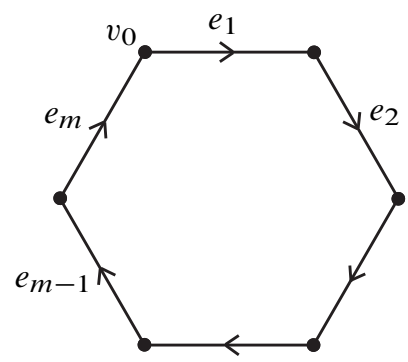

Figure 2. The cycle of $w$.

Notice that since $w$ is a reduced word, the graph $P\left(w, v_{0}\right)$ is well labeled. If $w$ is weakly cyclically reduced, then $C\left(w, v_{0}\right)$ is also well labeled.

Reciprocally, if $P=\left\{e_{1}, e_{2}, \ldots, e_{n}\right\}$ is a well-labeled path with $i\left(e_{1}\right)=v_{0}$, labeled by $l\left(e_{i}\right)=g_{i}$ for all $i$, then there exists a unique reduced word $w=g_{n} \ldots g_{1}$ such that $P\left(w, v_{0}\right)$ is $P$. If $C=\left\{e_{1}, e_{2}, \ldots, e_{n}\right\}$ is a well-labeled cycle with $t\left(e_{n}\right)=i\left(e_{1}\right)=v_{0}$, labeled by $l\left(e_{i}\right)=g_{i}$ for all $i$, then there exists a unique weakly cyclically reduced word $w=g_{n} \ldots g_{1}$ such that $C\left(w, v_{0}\right)$ is $C$.

Let $X$ be an infinite countable set. Let $\beta$ be a simply transitive permutation of $X$. We shall represent the $\beta$-action on $X$ as an infinite 2-regular well-labeled graph. The pre-graph $G_{0}$ is a labeled graph consisting of the set of vertices $V\left(G_{0}\right)=X$ and the set of edges $E\left(G_{0}\right)$ where for all $e \in E\left(G_{0}\right), l(e) \in\left\{\beta^{ \pm 1}\right\}$ and such that every vertex has exactly one entering edge and one leaving edge. One can imagine $G_{0}$ as the Cayley graph of $\mathbb{Z}$ with 1 as a generator.

Definition 3.4. An extension of $G_{0}$ is a well-labeled graph $G$ labeled by $\left\{\alpha^{ \pm 1}, \beta^{ \pm 1}\right\}$, containing $G_{0}$. We will denote it by $G_{0} \subset G$. 
In order to have a transitive action with some additional properties of the $\langle\alpha, \beta\rangle$ action on $X$, we shall extend $G_{0}$ by adding finitely many directed edges labeled by $\alpha$ on $G_{0}$ where the edges labeled by $\beta$ are already prescribed. In order that the added edges represent an action on $X$, we put the edges in such a way that the extended graph is well labeled, and moreover we put an additional edge labeled by $\alpha$ on every endpoint of the extended edges by $\alpha$; more precisely, if we have added $n$ edges labeled by $\alpha$ between $x_{0}, x_{1}, \ldots, x_{n}$ successively, we put an $\alpha$-edge from $x_{n}$ to $x_{0}$ to have a cycle consisting of $n+1$ edges (see Figure 3). On the points where no $\alpha$-edges are involved, we put a loop labeled by $\alpha$; this means that these points are the fixed points of $\alpha$. In the end, every point has a entering edge and a leaving edge labeled by $\alpha$ (the entering edge is equal to the leaving edge if the edge is a loop), so that the graph represents an $\langle\alpha, \beta\rangle$-action on $X$, and every $\alpha$-orbit is finite.

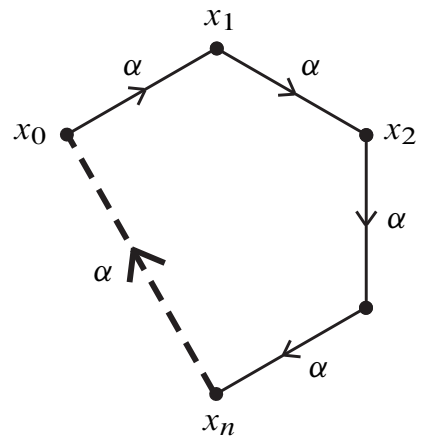

Figure 3. The $\alpha$-orbit of $x_{0}$ that has the size $n+1$.

Definition 3.5. Let $G, G^{\prime}$ be graphs labeled by $\left\{\alpha^{ \pm 1}, \beta^{ \pm 1}\right\}$. A homomorphism $f: G \rightarrow G^{\prime}$ is a map sending vertices to vertices, edges to edges, such that

- $f(i(e))=i(f(e))$ and $f(t(e))=t(f(e))$,

- $l(e)=l(f(e))$,

for all $e \in E(G)$.

If there exists an injective homomorphism $f: G \rightarrow G^{\prime}$, we say that $f$ is an embedding, and $G$ embeds in $G^{\prime}$. If there exists a bijective homomorphism $f: G \rightarrow$ $G^{\prime}$, we say that $f$ is an isomorphism, and $G$ is isomorphic to $G^{\prime}$.

Proposition 6. Let $w=w_{m} \ldots w_{1}$ be a reduced word on $\left\{\alpha^{ \pm 1}, \beta^{ \pm 1}\right\}$, and let $P\left(w, v_{0}\right)=\left\{e_{1}, \ldots, e_{m}\right\}$ be the path defined in Definition 3.2. There exists an extension $G$ of $G_{0}$ such that $P\left(w, v_{0}\right)$ embeds in $G$, and $P\left(w, v_{0}\right)$ is isomorphic to its image by the corresponding embedding. In particular, the image of $P\left(w, v_{0}\right)$ is a path in $G$.

Proof. It is enough to consider the case where $w=\alpha^{a_{2 n}} \beta^{b_{2 n-1}} \ldots \alpha^{a_{4}} \beta^{b_{3}} \alpha^{a_{2}} \beta^{b_{1}}$, with $m=\sum_{i=1}^{n}\left(\left|b_{2 i-1}\right|+\left|a_{2 i}\right|\right)$. Indeed, the other three cases follow from this case 
by taking $n$ large enough since we are treating all subwords of $w$. Let $N=\max _{j}\left|b_{j}\right|$. For $z \in X$, denote by $B_{N}(z)=\left\{\beta^{l} z \mid-N \leq l \leq N\right\}$ a segment in the $\beta$-orbit of $z$.

Choose $z_{0} \in X$. For all $1 \leq k \leq n$, we extend $G_{0}$ inductively by applying the following algorithm.

\section{Algorithm (A)}

(1) Let $z_{2 k-1}=\beta^{b_{2 k-1}} z_{2 k-2}$;

(2) choose $z_{2 k} \in X$ such that $B_{N}\left(z_{2 k}\right)$ is outside of the finite set of all used points;

(3) choose $\left|a_{2 k}\right|-1$ points $\left\{p_{1}^{\left(a_{2 k}\right)}, \ldots, p_{\left|a_{2 k}\right|-1}^{\left(a_{2 k}\right)}\right\}$ outside of the finite set of all points used so far;

(4) put the directed edges labeled by $\alpha^{\operatorname{sign}\left(a_{2 k}\right)}$ from

- $z_{2 k-1}$ to $p_{1}^{\left(a_{2 k}\right)}$;

- $p_{j}^{\left(a_{2 k}\right)}$ to $p_{j+1}^{\left(a_{2 k}\right)}$ for all $1 \leq j \leq\left|a_{2 k}\right|-2$;

- $p_{\left|a_{2 k}\right|-1}^{\left(a_{2 k}\right)}$ to $z_{2 k}$,

so that we have $\alpha^{a_{2 k}} z_{2 k-1}=z_{2 k}$.

In the end, we have added $\sum_{i=1}^{n}\left|a_{2 i}\right|$ new directed edges labeled by $\alpha$ (or $\alpha^{-1}$ ) on $G_{0}$ (see Figure 4). Let $G$ be the extended graph of $G_{0}$. In this construction, we have considered $|w|+1$ points $\left\{z_{0}, \beta^{\operatorname{sign}\left(b_{1}\right)} z_{0}, \beta^{2 \operatorname{sign}\left(b_{1}\right)} z_{0}, \ldots, \beta^{b_{1}} z_{0}=z_{1}\right.$, $\left.\alpha^{\operatorname{sign}\left(a_{2}\right)} \beta^{b_{1}} z_{0}, \ldots, \alpha^{a_{2}} \beta^{b_{1}} z_{0}=z_{2}, \ldots, w z_{0}\right\}$ in $X$ that are

$$
\left\{z_{0}, w_{1} z_{0}, w_{2} w_{1} z_{0}, \ldots, w z_{0}\right\}
$$

with $l\left(\left(w_{k-1} \ldots w_{1} z_{0}\right),\left(w_{k} w_{k-1} \ldots w_{1} z_{0}\right)\right)=w_{k}$, where $\left(p_{1}, p_{2}\right)$ symbolizes the edge $e$ with $i(e)=p_{1}$ and $t(e)=p_{2}$.

Now we define an embedding $f: P\left(w, v_{0}\right) \hookrightarrow G$ by

$$
\begin{aligned}
E\left(P\left(w, v_{0}\right)\right) & \rightarrow E(G), \\
e_{1}=\left(v_{0}, t\left(e_{1}\right)\right) & \mapsto\left(z_{0}, w_{1} z_{0}\right), \\
e_{k}=\left(i\left(e_{k}\right), t\left(e_{k}\right)\right) & \mapsto\left(w_{k-1} \ldots w_{1} z_{0}, w_{k} \ldots w_{1} z_{0}\right) \quad \text { for all } 2 \leq k \leq m .
\end{aligned}
$$

By construction, $P\left(w, v_{0}\right)$ is isomorphic to its image.

Proposition 7. Let $w=w_{m} \ldots w_{1}$ be a weakly cyclically reduced word on $\left\{\alpha^{ \pm 1}\right.$, $\left.\beta^{ \pm 1}\right\}$ with $w \notin\langle\beta\rangle$. Let $C\left(w, v_{0}\right)=\left\{e_{1}, \ldots, e_{m}\right\}$ be the cycle defined in Definition 3.3. There exists an extension $G$ of $G_{0}$ such that $C\left(w, v_{0}\right)$ embeds in $G$, and $C\left(w, v_{0}\right)$ is isomorphic to its image by the corresponding embedding. In particular, the image of $C\left(w, v_{0}\right)$ is a cycle in $G$.

Proof. It is enough to consider the case where $w=\alpha^{a_{2 n}} \beta^{b_{2 n-1}} \ldots \alpha^{a_{4}} \beta^{b_{3}} \alpha^{a_{2}} \beta^{b_{1}}$, with $m=\sum_{i=1}^{n}\left(\left|b_{2 i-1}\right|+\left|a_{2 i}\right|\right)$. Let $N=\max _{j}\left|b_{j}\right|$. 


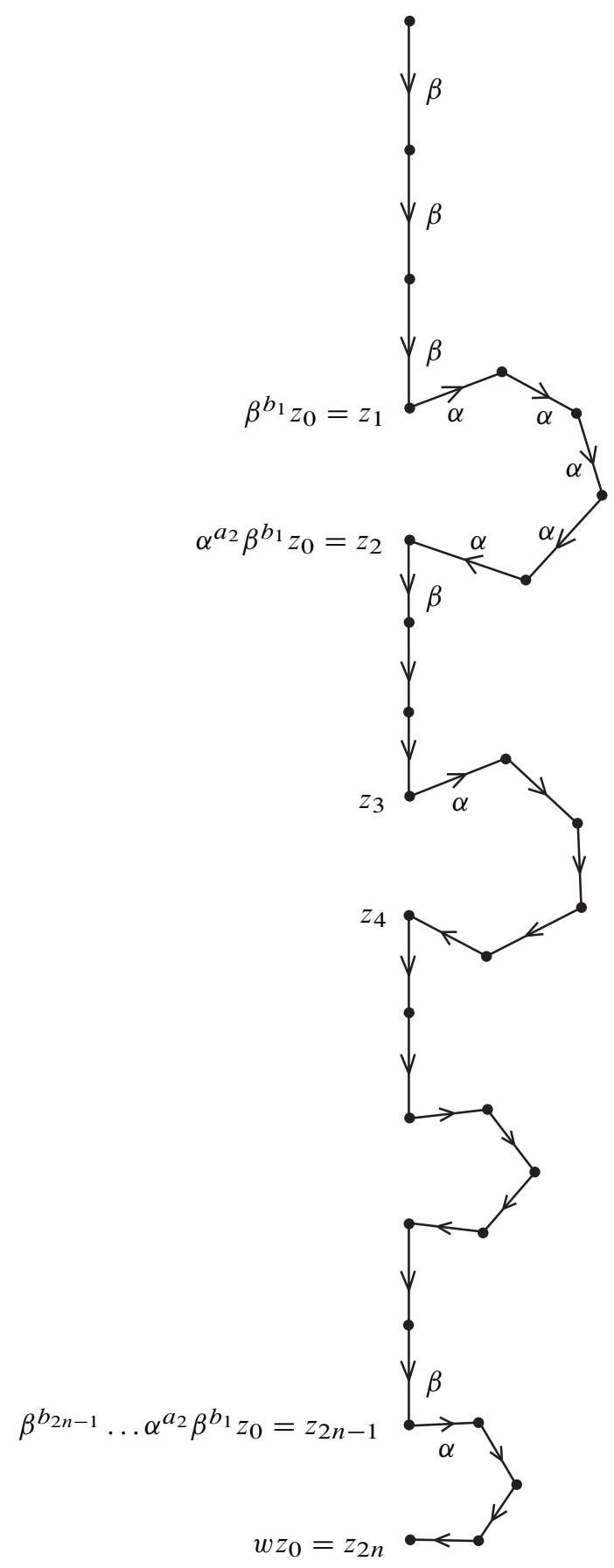

Figure 4. Construction of a path in $G$. 
Choose $z_{0} \in X$. We extend $G_{0}$ inductively by applying Algorithm (A) for $1 \leq$ $k \leq n-1$. Let $z_{2 n-1}=\beta^{b_{2 n-1}} z_{2 n-2}$. Choose $\left|a_{2 n}\right|-1$ points $\left\{p_{1}, \ldots, p_{\left|a_{2 n}\right|-1}\right\}$ outside of the finite set of all points used so far. Put the directed edges labeled by $\alpha^{\operatorname{sign}\left(a_{2 n}\right)}$ from

- $z_{2 n-1}$ to $p_{1}$;

- $p_{j}$ to $p_{j+1}$ for all $1 \leq j \leq\left|a_{2 n}\right|-2$;

- $p_{\left|a_{2 n}\right|-1}$ to $z_{0}$.

We define an embedding $f: C\left(w, v_{0}\right) \hookrightarrow G$ by

$$
\begin{aligned}
E\left(C\left(w, v_{0}\right)\right) & \rightarrow E(G), \\
e_{1}=\left(v_{0}, t\left(e_{1}\right)\right) & \mapsto\left(z_{0}, w_{1} z_{0}\right), \\
e_{k}=\left(i\left(e_{k}\right), t\left(e_{k}\right)\right) & \mapsto\left(w_{k-1} \ldots w_{1} z_{0}, w_{k} \ldots w_{1} z_{0}\right) \quad \text { for all } 2 \leq k \leq m-1, \\
e_{m}=\left(i\left(e_{m}\right), v_{0}\right) & \mapsto\left(w_{m-1} \ldots w_{1} z_{0}, z_{0}\right) .
\end{aligned}
$$

By construction, $C\left(w, v_{0}\right)$ is isomorphic to its image.

Corollary 8. Let $w$ be a reduced word. Let $F \subset G_{0}$ be a finite subset of $X$. There exists an extension $G$ of $G_{0}$ such that $P=P\left(w, v_{0}\right)$ embeds in $G$, the image $\bar{P}$ of $P$ is isomorphic to $P$, and the intersection of $\bar{P}$ and $F$ is empty. In addition, we can replace $P\left(w, v_{0}\right)$ by $C\left(w, v_{0}\right)$ if $w$ is weakly cyclically reduced and $w \notin\langle\beta\rangle$.

Proof. The construction of the extension consists of choosing some finite points in $X$. Therefore, it is enough to choose all considering points far enough outside of $F$.

3.1.2. Property (FF). Let $c=c_{m} \ldots c_{1}$ be a weakly cyclically reduced word such that $c \notin\langle\beta\rangle$. Let $w=w_{k} \ldots w_{1}$ be a reduced word such that $w \notin\langle c\rangle$. Let $C\left(c, v_{0}\right)$ be the cycle defined in Definition 3.3. Let $P\left(w, v_{0}\right)$ be the path defined in Definition 3.2 such that every vertex of $P\left(w, v_{0}\right)$ (other than $\left.v_{0}\right)$ is distinct from every vertex in $C\left(c, v_{0}\right)$. Let $w v_{0}$ be the endpoint of $P\left(w, v_{0}\right)$. Let $C\left(c, w v_{0}\right)$ be the cycle with $i\left(c_{1}\right)=t\left(c_{m}\right)=w v_{0}$ such that every vertex of $C\left(c, w v_{0}\right)$ (other than $\left.w v_{0}\right)$ is distinct from every vertex in $P\left(w, v_{0}\right) \cup C\left(c, v_{0}\right)$ (see Figure 5). Let us denote by $Q_{0}$ the union of $C\left(c, v_{0}\right), P\left(w, v_{0}\right)$ and $C\left(c, w v_{0}\right)$. In general, this finite labeled graph

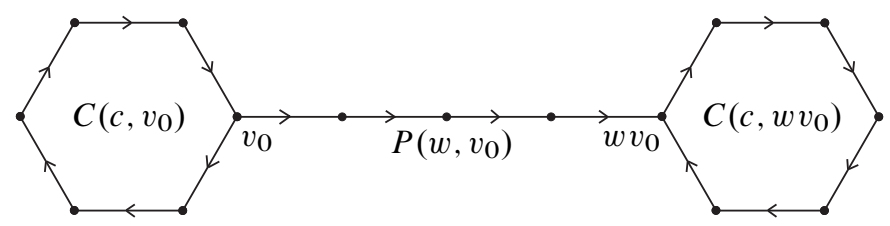

Figure 5. The graph $Q_{0}=C\left(c, v_{0}\right) \cup P\left(w, v_{0}\right) \cup C\left(c, w v_{0}\right)$. 
$Q_{0}$ is not well labeled. However, by identifying the successive edges with the same initial vertex and the same label, $Q_{0}$ becomes a well-labeled graph $Q$ (See Figure 6 for an example of the process).

$$
c=\alpha^{2} \beta \alpha, w=\alpha^{-1} \beta^{-1}
$$
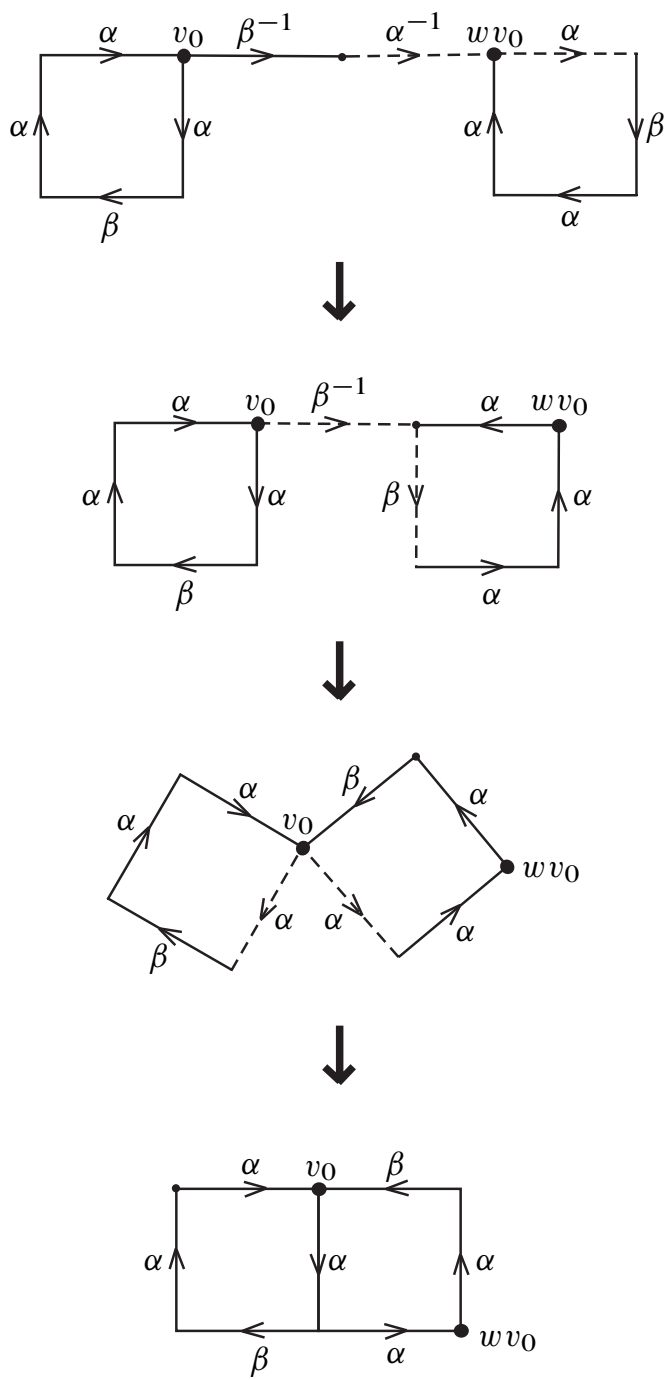

Figure 6. Example of gluing double edges.

In the end of the process of identification of "double edges", $Q$ has fewer edges than $Q_{0}$; however, the cycle $C\left(c, v_{0}\right)$ and $C\left(c, w v_{0}\right)$ are not modified, in the sense 
that the "shapes" of $C\left(c, v_{0}\right)$ and $C\left(c, w v_{0}\right)$ in $Q_{0}$ are the same as in $Q$. In other word, the quotient map $Q_{0} \rightarrow Q$ restricted to $C\left(c, v_{0}\right)$ and to $C\left(c, w v_{0}\right)$ is injective (each one separately).

By construction, in each process, the graph has the following property.

\section{Property (FF)}

(1) The starting point of $C\left(c, v_{0}\right)$ is equal to its endpoint which is $v_{0}$.

(2) The starting point of $P\left(w, v_{0}\right)$ is different from its endpoint.

(3) The starting point of $C\left(c, w v_{0}\right)$ is equal to its endpoint which is $w v_{0}$.

The acronym (FF) stands for "Faithfulness for $w$ and fixed points of $c$ ". Notice that (2) comes from the fact that $w \notin\langle c\rangle$. When this process is finished, $Q$ will be one of the following four types (Figure 7) of well-labeled graph satisfying the property (FF):

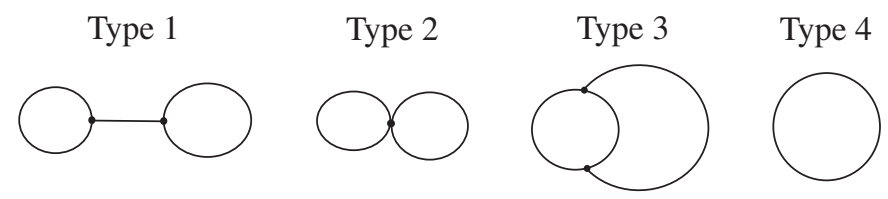

Figure 7. Four types of $Q$.

Proposition 9. For every one of the four types of well-labeled graph $Q=Q\left(c, w, v_{0}\right)$, there exists an extension $G$ of $G_{0}$ such that $Q$ embeds in $G$ and the image $Q\left(c, w, z_{0}\right)$ of $Q$ by the embedding has the property (FF), i.e., there exists $\alpha$ such that the word $w$ satisfies

$$
\left\{\begin{array}{l}
c z_{0}=z_{0}, \\
w z_{0} \neq z_{0}, \\
c w z_{0}=w z_{0},
\end{array}\right.
$$

where $z_{0}$ is the image of $v_{0}$ in $G$.

We have to prove that every cycle in each type in Figure 7 contains at least one directed edge labeled by $\alpha$ or $\alpha^{-1}$. This is clear for the type 1,2 and 4 since they have at most 2 cycles that represent $C\left(c, v_{0}\right)$ and $C\left(c, w v_{0}\right)$, and $c \notin\langle\beta\rangle$ by assumption. For type 3 , we can read around two subgraphs representing $C\left(c, v_{0}\right)$ and $P\left(w, v_{0}\right)^{-1} C\left(c, w v_{0}\right) P\left(w, v_{0}\right)$ from the vertex $v_{0}$. The labeling of the graph $P\left(w, v_{0}\right)^{-1} C\left(c, w v_{0}\right) P\left(w, v_{0}\right)$ is $w^{-1} c w$.

Let us recall the well-known theorem concerning the test for conjugacy of two words (see Theorem 1.3 in [12]).

Theorem 10. Two words in the free group $\mathbb{F}_{n}$ define conjugate elements of $\mathbb{F}_{n}$ if and only if their cyclic reductions in $\mathbb{F}_{n}$ are cyclic permutations of one another. 
Lemma 11. Let $c$ be a weakly cyclically reduced word such that $c \notin\langle\beta\rangle$. Let $w$ be a reduced word such that $w \notin\langle c\rangle$. If $c$ has the form $\gamma \beta^{l}$ with $\gamma \notin\langle\beta\rangle$, then $w^{-1} c w$ cannot be reduced to neither the form $\gamma \beta^{-k}$ nor the form $\gamma^{-1} \beta^{k}$ with $\operatorname{sign}(k)=\operatorname{sign}(l)$ for all $k \in \mathbb{Z}$.

Proof. Let $\gamma \beta^{l}$ with $\gamma=\gamma_{n} \ldots \gamma_{1} \notin\langle\beta\rangle$. By contradiction, let us suppose that $\gamma_{n} \ldots \gamma_{1} \beta^{l}$ is conjugate to $\gamma_{n} \ldots \gamma_{1} \beta^{-k}$ with $k, l>0$. Without loss of generality, we can suppose that $\gamma_{1}, \gamma_{n} \notin\left\{\beta^{ \pm 1}\right\}$. There are four types of cyclic permutations of $\gamma_{n} \ldots \gamma_{1} \beta^{l}$, which are $\gamma_{n} \ldots \gamma_{1} \beta^{l} ; \beta^{l} \gamma_{n} \ldots \gamma_{1} ; \beta^{l_{1}} \gamma_{n} \ldots \gamma_{1} \beta^{l_{2}}$ with $l_{1}+l_{2}=l$; and $\gamma_{p} \ldots \gamma_{1} \beta^{l} \gamma_{n} \ldots \gamma_{p+1}$ for a certain $1 \leq p \leq n$. Obviously, $\gamma_{n} \ldots \gamma_{1} \beta^{-k}$ cannot be of the first three types; so let us suppose that there exists $1 \leq p \leq n$ such that $\gamma_{p} \ldots \gamma_{1} \beta^{l} \gamma_{n} \ldots \gamma_{p+1}=\gamma_{n} \ldots \gamma_{1} \beta^{-l}$ (since the two conjugate elements have the same length). By identification of the $l^{\text {th }}$ letter on the right of the two words, we have $\beta^{-1}=\gamma_{p+l}=\gamma_{j}$, for every $j$ multiple of $p+l$ modulo $n+l$, so in particular $\beta^{-1}=\gamma_{n-p}$. However, by identifying the $(n-p+l)^{\text {th }}$ letter, which is $\beta$ for the left side, and $\gamma_{n-p}$ for the right side, we have $\beta=\gamma_{n-p}$ which contradicts with the first identification. The second case can be treated similarly.

Proof of Proposition 9. As we mentioned before, it remains to consider type 3.

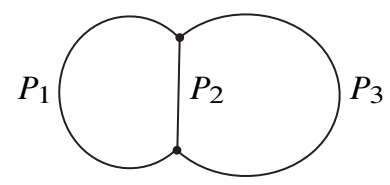

Figure 8. Type 3 of $Q$.

In this graph, there are three cycles $C=P_{1} \cup P_{2}, P_{2} \cup P_{3}$ and $P_{1} \cup P_{3}$.

Claim. If one of the three paths $P_{1}, P_{2}$ and $P_{3}$ has only edges labeled by $\beta^{ \pm 1}$, then the other two paths both contains edges labeled by $\alpha^{ \pm 1}$.

The claim allows to conclude. In fact, without loss of generality, suppose that $P_{1}$ has only edges labeled by $\beta^{ \pm 1}$ and $P_{2} \notin\langle\beta\rangle$ and $P_{3} \notin\langle\beta\rangle$. We first take an extension $G_{1} \supset G_{0}$ such that the image of $P_{1}$ is a path in $G_{1}$. Then we take an extension $G_{2} \supset G_{1}$ such that $P_{2}$ is a path in $G_{2}$ which connects the starting point and the endpoint of $P_{1}$ outside of the finite subset $P_{1}$; that is possible since the graph is well labeled and $P_{2}$ contains edges labeled by $\alpha$. Finally, we take an extension $G_{3} \supset G_{2}$ such that $P_{3}$ is a path in $G_{3}$ joining these two points outside of $P_{1} \cup P_{2}$.

We now prove the claim. Indeed, if two of these three paths were labeled by $\beta^{ \pm 1}$, then $c$ would be the form of $\gamma \beta^{l}$ up to cyclic permutation and $w^{-1} c w$ would be the form of $\gamma \beta^{-k}$ or $\gamma^{-1} \beta^{k}$ with $\operatorname{sign}(l)=\operatorname{sign}(k)$ up to cyclic permutation, which contradicts Lemma 11 . 
Corollary 12. Let $Q=Q\left(c, w, v_{0}\right)$ be a well-labeled graph. Let $F \subset G_{0}$ be a finite subset of $X$. There exists an extension $G$ of $G_{0}$ such that the image $Q\left(c, w, z_{0}\right)$ of $Q\left(c, w, v_{0}\right)$ in $G$ preserves the property $(\mathrm{FF})$, and the intersection of $Q\left(c, w, z_{0}\right)$ and $F$ is empty.

3.1.3. Proof of Proposition 1. Let $c=\alpha^{a_{1}} \beta^{b_{1}} \ldots \alpha^{a_{n}} \beta^{b_{n}}$ be a weakly cyclically reduced word on $\left\{\alpha^{ \pm 1}, \beta^{ \pm 1}\right\}$ (the other three types are similar). Let $w \in\langle\alpha, \beta\rangle \backslash\langle c\rangle$ be a reduced word on $\left\{\alpha^{ \pm 1}, \beta^{ \pm 1}\right\}$. We shall prove that the set

$$
\begin{gathered}
\mathcal{V}_{w}=\{\alpha \in \operatorname{Sym}(X) \mid \text { there exists a finite number of } x \in X \text { such that } \\
c x=x, c w x=w x \text { and } w x \neq x\}
\end{gathered}
$$

is meagre. For $K \subset X$ a finite subset of $X$, let

$$
V_{w, K}=\left\{\alpha \in \operatorname{Sym}(X) \mid\left(\operatorname{Fix}(c) \cap w^{-1} \operatorname{Fix}(c) \cap \operatorname{supp}(w)\right) \subseteq K\right\},
$$

where $\operatorname{supp}(w)=\{x \in X \mid w x \neq x\}$.

The set $V_{w, K}$ is closed since if $\alpha_{n}$ converges to $\alpha$, then $c\left(\alpha_{n}, \beta\right)$ converges to $c(\alpha, \beta)$ and $w\left(\alpha_{n}, \beta\right)$ converges to $w(\alpha, \beta)$. We shall prove that the interior of $V_{w, K}$ is empty.

Lemma 13. Let $\alpha^{\prime} \in \operatorname{Sym}(X)$ and $F \subset X$ be a finite subset of $X$. There exists $\alpha \in \operatorname{Sym}(X)$ such that $\left.\alpha\right|_{F}=\left.\alpha^{\prime}\right|_{F}$ and $\operatorname{supp}(\alpha) \subset F \cup \alpha^{\prime}(F)$.

Proof. We partition $F$ into finitely many pieces $F=\bigsqcup_{i=1}^{m} P_{i}$ according to the orbits of $\alpha^{\prime}$. If $\alpha^{\prime}\left(P_{i}\right)=P_{i}$, put $\left.\alpha\right|_{P_{i}}=\left.\alpha^{\prime}\right|_{P_{i}}$; if not, let $P_{i}=\left\{p_{i}, \alpha^{\prime}\left(p_{i}\right), \ldots, \alpha^{\prime k_{i}}\left(p_{i}\right)\right\}$ with $\alpha^{\prime k_{i}+1}\left(p_{i}\right) \notin F$. Then define $\left.\alpha\right|_{P_{i}}=\left.\alpha^{\prime}\right|_{P_{i}}$ and $\alpha\left(\alpha^{\prime k_{i}+1}\left(p_{i}\right)\right)=p_{i}$.

We see $X$ as the pre-graph $G_{0}$, where the $\beta^{ \pm 1}$-edges of $G_{0}$ are seen as the transitive action of $\beta^{ \pm 1}$ on $X$, which is fixed from the beginning.

Let $\alpha^{\prime} \in V_{w, K}$ and let $F \subset X$ be a finite subset of $X$. Let $Y=F \cup \alpha^{\prime}(F) \cup$ $K$ be a finite subset of $X$. We construct a well-labeled graph $Q\left(c, w, v_{0}\right)$ as in Section 3.1.2. We choose $z_{0} \notin Y$ and take $\alpha$ which is defined on $F$ as in Lemma 13, and which satisfies the property (FF) without touching any point of $Y$ (Corollary 12). Consequently, $\alpha \notin V_{w, K}$ and $\left.\alpha\right|_{F}=\left.\alpha^{\prime}\right|_{F}$.

3.1.4. Proof of Proposition 2. We want to prove that for all $k \in \mathbb{Z} \backslash\{0\}$, the set

$$
\mathcal{V}_{k}=\left\{\alpha \in \operatorname{Sym}(X) \mid c^{k}=\mathrm{Id}\right\}
$$

is closed and of empty interior.

Indeed, it is clearly closed. Moreover, let $\alpha^{\prime} \in \mathcal{V}_{k}$ and let $F \subset X$ be a finite subset of $X$. Let $P\left(c^{k}, v_{0}\right)$ be the path defined in Definition 3.2. We choose $z_{0} \notin$ $F \cup \alpha^{\prime}(F)=: Y$ and take $\alpha$, which is defined on $F$ as in Lemma 13, such that $P\left(c^{k}, z_{0}\right)$ is a path in $X$ not touching any point of $Y$. Consequently, $\alpha \notin \mathcal{V}_{k}$ and $\left.\alpha\right|_{F}=\left.\alpha^{\prime}\right|_{F}$. 
3.2. Proof of Proposition 3. Let $c$ be a special word. Let $\left\{A_{n}\right\}_{n \geq 1}$ be a pairwise disjoint Følner sequence for $\beta$. Let $\left\{\varepsilon_{l}\right\}_{l \geq 1}>0$ be a sequence tending to 0 . Let us write

$$
\begin{gathered}
u_{3}=\bigcap_{l} \bigcap_{N \in \mathbb{N}}\left\{\alpha \in \operatorname{Sym}(X) \mid \text { there exists } k \geq N \text { such that } A_{k} \subset \operatorname{Fix}(c)\right. \\
\text { and } \left.\left|A_{k} \triangle \alpha A_{k}\right|<\varepsilon_{l}\left|A_{k}\right|\right\} .
\end{gathered}
$$

Set $\varepsilon_{l}=\varepsilon$. We want to prove that the set

$$
\mathcal{V}_{N}:=\left\{\alpha \in \operatorname{Sym}(X) \mid \text { for all } k \geq N, A_{k} \nsubseteq \operatorname{Fix}(c) \text { or }\left|A_{k} \Delta \alpha A_{k}\right| \geq \varepsilon\left|A_{k}\right|\right\}
$$

is closed and of empty interior. We treat the case $c=\alpha^{a_{1}} \beta^{b_{1}} \ldots \alpha^{a_{n}} \beta^{b_{n}}$ (the other three types are similar). Let $M=\max _{j}\left|b_{j}\right|$ and set

$$
E_{k}:=\cup_{i=-M}^{M} \beta^{i}\left(A_{k}\right)
$$

a finite set of $X$.

$\mathcal{V}_{N}$ is closed. Since $\mathcal{V}_{N}=\bigcap_{k \geq N} \mathcal{V}_{N, k}$, where

$$
\mathcal{V}_{N, k}:=\left\{\alpha \in \operatorname{Sym}(X) \mid A_{k} \not \subset \operatorname{Fix}(c) \text { or }\left|A_{k} \Delta \alpha A_{k}\right| \geq \varepsilon\left|A_{k}\right|\right\},
$$

it is enough to prove that $\mathcal{V}_{N, k}$ is closed. So let $\left\{\alpha_{n}\right\}_{n \geq 1}$ be a sequence in $\mathcal{V}_{N, k}$ that converges to $\alpha \in \operatorname{Sym}(X)$. Since $E_{k}$ is finite, there exists $n_{0}$ such that $\left.\alpha\right|_{E_{k}}=\left.\alpha_{n}\right|_{E_{k}}$ for all $n \geq n_{0}$. Therefore, $\alpha \in \mathcal{V}_{N, k}$ because $A_{k} \subset E_{k}$.

$\mathcal{V}_{N}$ is of empty interior. Let us distinguish two cases:

First, suppose that $S(\alpha)=S(\beta)=0$. Let $\alpha^{\prime} \in \mathcal{V}_{N}$. Let $F \subset X$ be a finite subset of $X$. We choose $m \gg N$ such that $\left(F \cup \alpha^{\prime}(F)\right) \cap E_{m}=\emptyset$. We define $\left.\alpha\right|_{E_{m}}=$ Id and $\left.\alpha\right|_{F}=\left.\alpha^{\prime}\right|_{F}$. Then $A_{m} \subset \operatorname{Fix}(c)$ since $S(\beta)=0$, and $\left|A_{m} \Delta \alpha A_{m}\right|=0$ since $\alpha\left(A_{m}\right)=A_{m}$. So $\alpha \notin \mathcal{V}_{N}$.

Second, suppose that $S(\alpha)$ divides $S(\beta)$. Let $\alpha^{\prime} \in \mathcal{V}_{N}$. Let $F \subset X$ be a finite subset of $X$. We choose $m \gg N$ such that $\left(F \cup \alpha^{\prime}(F)\right) \cap E_{m}=\emptyset$ and $\left|A_{m} \Delta \beta^{-\frac{S(\beta)}{S(\alpha)}}\left(A_{m}\right)\right|<\varepsilon\left|A_{m}\right|$; this is possible as $\left\{A_{m}\right\}$ is a Følner sequence for $\beta$. We define

$$
\alpha(x)=\beta^{-\frac{S(\beta)}{S(\alpha)}}(x) \text { for all } x \in E_{m},
$$

and $\left.\alpha\right|_{F}=\left.\alpha^{\prime}\right|_{F}$. Then

$$
c(x)=\beta^{-\frac{S(\beta)}{S(\alpha)} a_{1}} \beta^{b_{1}} \ldots \beta^{-\frac{S(\beta)}{S(\alpha)} a_{n}} \beta^{b_{n}}(x)=\beta^{-\frac{S(\beta)}{S(\alpha)} S(\alpha)} \beta^{S(\beta)}(x)=x,
$$

for all $x \in E_{m}$. In particular, $A_{m} \subset \operatorname{Fix}(c)$. In addition,

$$
\left|A_{m} \Delta \alpha A_{m}\right|=\left|A_{m} \Delta \beta^{-\frac{S(\beta)}{S(\alpha)}}\left(A_{m}\right)\right|<\varepsilon\left|A_{m}\right|,
$$

so $\alpha \notin \mathcal{V}_{N}$. 
3.3. Proof of Proposition 4. The proof follows from the three claims:

Claim 1. Let $G$ be a group and $H<G$ be a finite index subgroup of $G$. Then, for all $g \in G$, there exists $n \geq 1$ such that $g^{n} \in H$.

Indeed, let $N$ be the core of $H$, that is $N=\bigcap_{x \in G} x^{-1} H x \subset H$. The subgroup $N$ is a finite index normal subgroup of $G$. Then $g^{m} \in N$, where $m=[G: N]$, for all $g \in G$.

Claim 2. The set

$U_{5}=\left\{\alpha \in \operatorname{Sym}(X) \mid\right.$ the $\left\langle\alpha^{n}, \beta^{m}\right\rangle$-action on $X$ is transitive for all $\left.n, m \in \mathbb{Z} \backslash\{0\}\right\}$ is in $U_{4}$.

Indeed, let $\alpha \in \mathcal{U}_{5}$. Let $H<\langle\alpha, \beta\rangle$ be a finite index subgroup. Then by Claim 1 there exist $n_{0}, m_{0}$ such that $\alpha^{n_{0}}$ and $\beta^{m_{0}}$ are in $H$, so $\left\langle\alpha^{n_{0}}, \beta^{m_{0}}\right\rangle<H$. Since the $\left\langle\alpha^{n_{0}}, \beta^{m_{0}}\right\rangle$-action on $X$ is transitive by hypothesis, the $H$-action on $X$ is also transitive.

Claim 3. The set $\mathcal{U}_{4}$ is generic in $\operatorname{Sym}(X)$.

It is enough to prove that the set $U_{5}$ is generic since $U_{5} \subset U_{4}$. So let us prove that the set $\mathcal{V}_{n, m}=\left\{\alpha \in \operatorname{Sym}(X) \mid\right.$ the $\left\langle\alpha^{n}, \beta^{m}\right\rangle$-action on $\mathrm{X}$ is not transitive $\}$ is closed for all $n$ and $m$, and has empty interior.

$\mathcal{V}_{n, m}$ is closed.

$$
\begin{aligned}
\mathcal{V}_{n, m} & =\left\{\alpha \in \operatorname{Sym}(X) \mid \exists x, y \in X \text { such that } w x \neq y \text { for all } w \in\left\langle\alpha^{n}, \beta^{m}\right\rangle\right\} \\
& =\left\{\alpha \in \operatorname{Sym}(X) \mid \exists\left(x_{i}, x_{j}\right) \in S \times S \text { such that } w x_{i} \neq x_{j}\right. \\
& =\bigcup_{\left(x_{i}, x_{j}\right) \in S \times S}\left\{\alpha \in \operatorname{Sym}(X) \mid w x_{i} \neq x_{j} \text { for all } w \in\left\langle\alpha^{n}, \beta^{m}\right\rangle\right\}
\end{aligned}
$$

where $S$ is a finite family of representatives for $\beta^{m}$-orbits. It is clear that the set $\left\{\alpha \in \operatorname{Sym}(X) \mid w x_{i} \neq x_{j}\right.$ for all $\left.w \in\left\langle\alpha^{n}, \beta^{m}\right\rangle\right\}$ is closed. So $\mathcal{V}_{n, m}$ is closed as a finite union of closed sets.

$\mathcal{V}_{n, m}$ is of empty interior. Let $\alpha^{\prime} \in \mathcal{V}_{n, m}$ and let $F \subset X$ be a finite subset of $X$. Let $Y:=F \cup \alpha^{\prime}(F)$ be a finite subset of $X$. We choose representatives for $\beta^{m}$-orbits outside of $Y$, and form a finite family $S=\left\{x_{1}, \ldots, x_{m}\right\}$ of $X$; this is possible since the $\beta^{m}$-orbits are infinite. We define $\alpha$ on $F$ as in Lemma 13. Inductively on $1 \leq i \leq m-1$, in each $\beta^{m}$-orbit $O\left(x_{i}\right)$ of $x_{i}$, we choose $n-1$ points $\left\{p_{i, 1}, p_{i, 2}\right.$, $\left.\ldots, p_{i, n-1}\right\}$ outside of $Y$ and define

- $\alpha\left(x_{i}\right)=p_{i, 1}$;

- $\alpha\left(p_{i, j}\right)=p_{i, j+1}$, for all $1 \leq j \leq n-2$;

- $\alpha\left(p_{i, n-1}\right)=x_{i+1}$.

Then, in $O\left(x_{m}\right)$, we choose $n-1$ points $\left\{p_{m, 1}, \ldots, p_{m, n-1}\right\}$ outside of $Y$ and define

- $\alpha\left(x_{m}\right)=p_{m, 1}$; 
- $\alpha\left(p_{m, j}\right)=p_{m, j+1}$ for all $1 \leq j \leq n-2$;

- $\alpha\left(p_{m, n-1}\right)=x_{1}$.

By construction, $\alpha^{n}\left(x_{i}\right)=x_{i+1}$ for all $1 \leq i \leq m-1$, and $\alpha^{n}\left(x_{m}\right)=x_{1}$, so the $\left\langle\alpha^{n}, \beta^{m}\right\rangle$-action is transitive.

\section{Construction of $\mathbb{F}_{2} * \mathbb{Z} \mathbb{F}_{2}$}

Let $X$ be a countable infinite set. Let $c=c(\alpha, \beta)$ be a special word. Let $G:=\mathbb{F}_{2}=$ $\langle\alpha, \beta\rangle$ be constructed as in Section 3. Let $\left\{A_{n}\right\}_{n=1}^{\infty}$ be a Følner sequence such that $c\left(A_{n}\right)=A_{n}$ for all $n \geq 1$. Let $Z_{c}=\{\sigma \in \operatorname{Sym}(X) \mid \sigma c=c \sigma\}$ be the centralizer of $c$. Let $\alpha^{\prime}=\sigma^{-1} \alpha \sigma, \beta^{\prime}=\sigma^{-1} \beta \sigma$, and let $H:=\left\langle\alpha^{\prime}, \beta^{\prime}\right\rangle$. Let $A=\langle c\rangle$ be the subgroup of $G$ generated by $c$. We consider $\mathbb{F}_{2} * \mathbb{Z} \mathbb{F}_{2}=G *_{A} H$ the amalgamated free product of $G$ and $H$ along $A$. For all $\sigma \in Z$, the action of $G *_{A} H$ on $X$ is given by $g \cdot x=g(\alpha, \beta) x=g x$, and $h \cdot x=h\left(\alpha^{\prime}, \beta^{\prime}\right) x=\sigma^{-1} h(\alpha, \beta) \sigma x=\sigma^{-1} h \sigma x$ for all $g \in G$ and $h \in H$.

Lemma 14. The set $Z_{c}$ is closed in $\operatorname{Sym}(X)$. In particular, $Z_{c}$ is a Baire space.

Proof. The map $p: \operatorname{Sym}(X) \rightarrow \operatorname{Sym}(X), \sigma \mapsto[\sigma, c]$, is continuous. So $Z_{c}=$ $p^{-1}\{\mathrm{Id}\}$ is closed since $\{\mathrm{Id}\}$ is closed in $\operatorname{Sym}(X)$.

Proposition 15. The set

$$
\mathcal{O}_{1}=\left\{\sigma \in Z_{c} \mid \text { the action of } G *_{A} H \text { on } X \text { is faithful }\right\}
$$

is generic in $Z_{c}$.

Proof. For all $w \in G *_{A} H$, let us denote by $w^{\sigma}$ the corresponding element of $\operatorname{Sym}(X)$ given by the above action, i.e., if $w=a g_{n} h_{n} \ldots g_{1} h_{1}$, with $a \in A, g_{i} \neq e \in G \backslash A$ and $h_{i} \neq e \in H \backslash A$ for all $i$, then

$$
w^{\sigma}=a g_{n} \sigma^{-1} h_{n} \sigma \ldots g_{1} \sigma^{-1} h_{1} \sigma .
$$

We want to prove that the set

$$
\mathcal{O}_{1}=\bigcap_{w \neq e \in G *_{A} H}\left\{\sigma \in Z_{c} \mid \text { there exists } x \in X \text { such that } w^{\sigma} x \neq x\right\}
$$

is generic in $Z_{c}$. Therefore, we shall prove that the set

$$
\mathcal{V}_{w}=\left\{\sigma \in Z_{c} \mid w^{\sigma}=\operatorname{Id}_{X}\right\}
$$

is closed and of empty interior in $Z_{c}$.

The set $\mathcal{V}_{w}$ is closed in $Z_{c}$ because the map $Z_{\sigma} \rightarrow \operatorname{Sym}(X), \sigma \mapsto w^{\sigma}$, is continuous. 
To see that the set $\mathcal{V}_{w}$ is of empty interior, let $\sigma^{\prime} \in \mathcal{V}_{w}$, and let $F \subset X$ be a finite subset of $X$. Notice that if $F=F_{1} \sqcup F_{2}$ with $F_{1} \subset \operatorname{Fix}(c)$ and $F_{2} \cap \operatorname{Fix}(c)=\emptyset$, then $\sigma^{\prime}\left(F_{1}\right) \subset \operatorname{Fix}(c)$ and $\sigma^{\prime}\left(F_{2}\right) \cap \operatorname{Fix}(c)=\emptyset$ because $\sigma^{\prime}(\operatorname{Fix}(c))=\operatorname{Fix}(c)$ for all $\sigma^{\prime} \in Z_{c}$. So we define $\left.\sigma\right|_{F_{1}}=\left.\sigma^{\prime}\right|_{F_{1}}$ as in Lemma 13, and $\left.\sigma\right|_{X \backslash \operatorname{Fix}(c)}=\left.\sigma^{\prime}\right|_{X \backslash \operatorname{Fix}(c)}$. Therefore, we have defined $\sigma$ on $Y:=\left(F \cup \sigma^{\prime}(F)\right) \cup(X \backslash \operatorname{Fix}(c))$, and $\left.\sigma\right|_{Y}$ commutes with $\left.c\right|_{Y}$. Let us now define $\sigma$ on $X \backslash Y$ in a way that $\sigma \in Z_{c} \backslash \mathcal{V}_{w}$. For all $g \in G \backslash A$ and $h \in H \backslash A$, let

$$
\begin{aligned}
& \hat{g}=\{x \in X \mid \operatorname{cx}=x, \operatorname{cg} x=g x \text { and } g x \neq x\}, \\
& \hat{h}=\{x \in X \mid \operatorname{co}=x, \operatorname{ch} x=h x \text { and } h x \neq x\} .
\end{aligned}
$$

Recall that we are considering the word $w^{\sigma}=a g_{n} \sigma^{-1} h_{n} \sigma \ldots g_{1} \sigma^{-1} h_{1} \sigma$. Choose any $x_{0} \in X \backslash Y$. By induction on $1 \leq i \leq n$, we choose $x_{4 i-3} \in \hat{h_{i}}$ such that $x_{4 i-3}$ is different from the finite set of points $x_{1}, \ldots, x_{4 i-4}$ chosen until the (i-1)-th step. This is possible since $\hat{h_{i}}$ is infinite by Proposition 1 . Then we define $\sigma x_{4 i-4}:=x_{4 i-3}$ and $\sigma x_{4 i-3}:=x_{4 i-4}$. This is well defined because $x_{4 i-4}, x_{4 i-3} \in \operatorname{Fix}(c)$. We set $h_{i} x_{4 i-3}=: x_{4 i-2}$ which is different from $x_{4 i-3}$ and which is fixed by $c$, by definition of $\hat{h_{i}}$. We choose $x_{4 i-1} \in \hat{g_{i}}$ such that $x_{4 i-1}$ is different from the finite set of points chosen so far. This is again possible since $\widehat{g}_{i}$ is infinite (Proposition 1). Then we define $\sigma x_{4 i-2}:=x_{4 i-1}$ and $\sigma x_{4 i-1}:=x_{4 i-2}$. This is also well defined because $x_{4 i-2}, x_{4 i-1} \in \operatorname{Fix}(c)$. We finally set $g_{i} x_{4 i-1}=: x_{4 i}$. By construction, the $4 n$ points defined by the subwords on the right of $w^{\sigma}$ are all distinct. In particular, $w^{\sigma} x_{0}=a x_{4 n}=x_{4 n} \neq x_{0}$. Besides, this construction works also for the other three types of word $w$ since we are treating all subwords of $w$. At last, if $w=g \in G \backslash\{\mathrm{Id}\}$, then there exists $x \in X$ such that $g x \neq x$ since $G$ acts faithfully on $X$. Therefore, $\sigma$ constructed in this way is beautifully in $Z_{c} \backslash \mathcal{V}_{w}$ and $\left.\sigma^{\prime}\right|_{F}=\left.\sigma\right|_{F}$.

Proposition 16. The set

$$
\begin{gathered}
\mathcal{O}_{2}=\left\{\sigma \in Z_{c} \mid \text { there exists a subsequence }\left\{A_{n_{k}}\right\}_{k \geq 1} \text { of }\left\{A_{n}\right\}_{n \geq 1}\right. \\
\text { such that } \left.\sigma\left(A_{n_{k}}\right)=A_{n_{k}} \text { for all } k \geq 1\right\}
\end{gathered}
$$

is generic in $Z_{c}$.

Proof. We want to prove that the set

$$
\mathcal{O}_{2}=\bigcap_{N \in \mathbb{N}}\left\{\sigma \in Z_{c} \mid \text { there exists } n \geq N \text { such that } \sigma\left(A_{n}\right)=A_{n}\right\}
$$

is generic in $Z_{c}$. So we shall prove that the set

$$
\mathcal{V}_{N}=\left\{\sigma \in Z_{c} \mid \sigma\left(A_{n}\right) \neq A_{n} \text { for all } n \geq N\right\}
$$

is closed and of empty interior in $Z_{c}$. 
$\mathcal{V}_{N}$ is closed. It is enough to prove that the set

$$
V_{n, N}=\left\{\sigma \in Z_{c} \mid \sigma\left(A_{n}\right) \neq A_{n}\right\}
$$

is closed since $\mathcal{V}_{N}=\bigcap_{n \geq N} V_{n, N}$. Let $\left\{\sigma_{m}\right\}_{m \geq 1} \subset V_{n, N}$ be a sequence converging to $\sigma$ in $Z_{c}$. Since $A_{n}$ is finite, there exists $m_{0}$ such that $\sigma_{m}\left(A_{n}\right)=\sigma\left(A_{n}\right)$ for all $m \geq m_{0}$. Thus we have $\sigma\left(A_{n}\right) \neq A_{n}$ since $\sigma_{m}\left(A_{n}\right) \neq A_{n}$.

$\mathcal{V}_{N}$ is of empty interior. Let $\sigma^{\prime} \in \mathcal{V}_{N}$ and let $F \subset X$ be a finite subset of $X$. Let $Y:=\left(F \cup \sigma^{\prime}(F)\right) \cup(X \backslash \operatorname{Fix}(c))$. Since $A_{n} \subset \operatorname{Fix}(c)$ (Proposition 3), there exists $n \geq N$ such that $A_{n} \cap Y=\emptyset$. We take then $\sigma \in Z_{c}$ which fixes $A_{n}$ and $\left.\sigma\right|_{Y}=\left.\sigma^{\prime}\right|_{Y}$. Therefore, $\sigma \in Z_{c} \backslash \mathcal{V}_{N}$ and $\left.\sigma\right|_{F}=\left.\sigma^{\prime}\right|_{F}$.

Let $\sigma \in \mathcal{O}_{1} \cap \mathcal{O}_{2}$. Let $\left\{A_{n_{k}}\right\}_{k \geq 1}$ be a subsequence of $\left\{A_{n}\right\}_{n \geq 1}$ such that $\sigma\left(A_{n_{k}}\right)=$ $A_{n_{k}}$ for all $k \geq 1$. We claim that $\left\{A_{n_{k}}\right\}_{k \geq 1}$ is a Følner sequence for $G *_{A} H$. Indeed, for all $g \in G$ and for all $h \in H$, we have

$$
\begin{aligned}
\lim _{k \rightarrow \infty} \frac{\left|A_{n_{k}} \Delta g \cdot A_{n_{k}}\right|}{\left|A_{n_{k}}\right|} & =\lim _{k \rightarrow \infty} \frac{\left|A_{n_{k}} \Delta g(\alpha, \beta) A_{n_{k}}\right|}{\left|A_{n_{k}}\right|}=0, \\
\lim _{k \rightarrow \infty} \frac{\left|A_{n_{k}} \Delta h \cdot A_{n_{k}}\right|}{\left|A_{n_{k}}\right|} & =\lim _{k \rightarrow \infty} \frac{\left|A_{n_{k}} \Delta h\left(\alpha^{\prime}, \beta^{\prime}\right) A_{n_{k}}\right|}{\left|A_{n_{k}}\right|} \\
& =\lim _{k \rightarrow \infty} \frac{\left|A_{n_{k}} \Delta \sigma^{-1} h(\alpha, \beta) \sigma A_{n_{k}}\right|}{\left|A_{n_{k}}\right|} \\
& =\lim _{k \rightarrow \infty} \frac{\left|\sigma A_{n_{k}} \Delta h(\alpha, \beta) \sigma A_{n_{k}}\right|}{\left|A_{n_{k}}\right|} \\
& =\lim _{k \rightarrow \infty} \frac{\left|A_{n_{k}} \Delta h(\alpha, \beta) A_{n_{k}}\right|}{\left|A_{n_{k}}\right|}=0,
\end{aligned}
$$

since $\left\{A_{n_{k}}\right\}$ is Følner for $G$ and $\sigma\left(A_{n_{k}}\right)=A_{n_{k}}$. Therefore, we have:

Theorem 17. There exists a transitive, faithful and amenable action of the group $\langle\alpha, \beta\rangle *\langle c\rangle\left\langle\alpha^{\prime}, \beta^{\prime}\right\rangle$ on $X$.

Lemma 18. Let $c=c(\alpha, \beta)$ be any word (not necessarily special) on $\left\{\alpha^{ \pm 1}, \beta^{ \pm 1}\right\}$. There exists an automorphism a of $\mathbb{F}_{2}$ such that $a(c)$ is a special word.

Proof. Let us recall some properties of automorphisms of free groups. The reader can find more details in [11]. Let $\mathbb{F}_{n}$ be a free group with a finite basis $X$ of $n$ elements. We consider the following endomorphisms of $\mathbb{F}_{n}$. For any $x \in X$, let $\varphi_{x}$ be the endomorphism defined by $\varphi_{x}: x \mapsto x^{-1}, y \mapsto y$, for all $y \in X \backslash\{x\}$. For any $x \neq y \in X$, let $\psi_{x y}: x \mapsto x y, z \mapsto z$, for all $z \in X \backslash\{x\}$. In both cases, the image of $X$ is another basis for $\mathbb{F}_{n}$, and $\varphi_{x}$ and $\psi_{x y}$ are automorphisms of $\mathbb{F}_{n}$, called the Nielsen generators for $\operatorname{Aut}\left(\mathbb{F}_{n}\right)$, and they generate $\operatorname{Aut}\left(\mathbb{F}_{n}\right)$. Let $\mathbb{F}_{n} / \mathbb{F}_{n}^{\prime} \simeq \mathbb{Z}^{n}$ be 
the abelianization of $\mathbb{F}_{n}$. We have $\operatorname{Aut}\left(\mathbb{Z}^{n}\right) \simeq \mathrm{GL}_{n}(\mathbb{Z})$. The Nielsen generators for $\operatorname{Aut}\left(\mathbb{F}_{n}\right)$ induce the following generators for $\operatorname{Aut}\left(\mathbb{Z}^{n}\right)$ :

$$
\begin{array}{rlrl}
\bar{\varphi}_{x}: x & \mapsto-x, & y & \mapsto y \text { for all } y \in X \backslash\{x\} ; \\
\bar{\psi}_{x y}: x & \mapsto x+y, & z \mapsto z \text { for all } z \in X \backslash\{x\} .
\end{array}
$$

Thus, we conclude that the natural maps from $\operatorname{Aut}\left(\mathbb{F}_{n}\right)$ into $\operatorname{Aut}\left(\mathbb{Z}^{n}\right)$ is an epimorphism. Notice that for a word $c$ to be a special word depends only on its image in $\mathbb{Z}^{2}$. Therefore, in order to prove the lemma, it is enough to find a matrix $M \in \mathrm{GL}_{2}(\mathbb{Z})$ such that the exponent sum $S(\alpha)^{\prime}:=S_{a(c)}(\alpha)$ of exponents of $\alpha$ in the word $a(c)$ divides the exponent sum $S(\beta)^{\prime}:=S_{a(c)}(\beta)$ of exponents of $\beta$ in the word $a(c)$, where $a \in \operatorname{Aut}\left(\mathbb{F}_{2}\right)$ is a reciprocal image of $M$ by the epimorphism $\operatorname{Aut}\left(\mathbb{F}_{2}\right) \rightarrow \operatorname{Aut}\left(\mathbb{Z}^{2}\right)$. In fact, once we have $c=c(\alpha, \beta)$ with $S(\alpha)$ dividing $S(\beta)$, we can obtain a weakly cyclically reduced word by conjugating $c$, and the conjugation is an automorphism of $\mathbb{F}_{2}$.

If $S(\beta)=0, c$ is already a special word. If $S(\alpha)=0$ and $S(\beta) \neq 0$, then we apply the matrix $\left(\begin{array}{ll}0 & 1 \\ 1 & 0\end{array}\right) \in \mathrm{GL}_{2}(\mathbb{Z})$ which exchanges $S(\alpha)$ and $S(\beta)$. So suppose that $S(\alpha) \neq 0 \neq S(\beta)$. Let $d=\operatorname{gcd}(S(\alpha), S(\beta))$ be the greatest common divisor of $S(\alpha)$ and $S(\beta)$. By Bézout's identity, there exist relatively prime integers $p, q$ such that $p S(\alpha)+q S(\beta)=d$. Since $\operatorname{gcd}(p,-q)=1$, there exist $r, t$ such that $r p-t q=1$

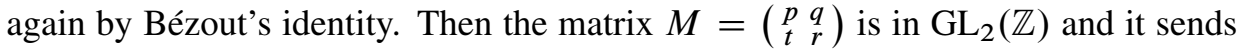
$\left(\begin{array}{l}S(\alpha) \\ S(\beta)\end{array}\right)$ to $\left(\begin{array}{c}d \\ t S(\alpha)+r S(\beta)\end{array}\right)$. Therefore, $S(\alpha)^{\prime}=d$ divides $S(\beta)^{\prime}=t S(\alpha)+r S(\beta)$.

From Theorem 17 and the previous lemma, we have:

Theorem 19. Let $c=c(\alpha, \beta)$ be any word on $\left\{\alpha^{ \pm 1}, \beta^{ \pm 1}\right\}$. Then the group $\langle\alpha, \beta\rangle *\langle c\rangle$ $\left\langle\alpha^{\prime}, \beta^{\prime}\right\rangle$ admits a transitive, faithful and amenable action.

A result of G. Baumslag [4] shows that these groups are residually finite.

Furthermore, let $H$ be a finite index subgroup of $\mathbb{F}_{2} * \mathbb{Z} \mathbb{F}_{2}$. Then $K:=H \cap \mathbb{F}_{2}$ is a finite index subgroup of $\mathbb{F}_{2}$ so that the $H$-action on $X$ is transitive since the $K$-action is transitive by Proposition 4. Therefore we have:

Theorem 20. For any finite index subgroup $H$ of $\langle\alpha, \beta\rangle *_{\langle c\rangle}\left\langle\alpha^{\prime}, \beta^{\prime}\right\rangle, H$ admits a transitive, faithful and amenable action.

\section{Applications}

Let us recall the class of all countable groups that appeared in [7]:

$\mathcal{A}=\{G$ countable $\mid G$ admits a faithful transitive amenable action $\}$. 
Let $\Sigma_{g}$ be a closed oriented surface of genus $g \geq 2$. It is well known that the fundamental group $\Gamma_{g}=\pi_{1}\left(\Sigma_{g}\right)$ of $\Sigma_{g}$ has a presentation

$$
\pi_{1}\left(\Sigma_{g}\right)=\left\langle a_{1}, b_{1}, \ldots, a_{g}, b_{g} \mid \prod_{i=1}^{g}\left[a_{i}, b_{i}\right]\right\rangle .
$$

In particular, we have $\pi_{1}\left(\Sigma_{2}\right)=\left\langle a_{1}, b_{1}\right\rangle *\langle c\rangle\left\langle a_{2}, b_{2}\right\rangle$ where $c=\left[a_{1}, b_{1}\right]=\left[a_{2}, b_{2}\right]$. Therefore, $\pi_{1}\left(\Sigma_{2}\right) \in \mathcal{A}$ by Theorem 19 (or already by Theorem 17 since $c=\left[a_{1}, b_{1}\right]$ is a special word). Now, let $\Sigma_{g}$ be a closed oriented surface of genus $g \geq 3$. Viewing $\Sigma_{g}$ as $(g-1)$ tori glued on a central one, the cyclic group $\mathbb{Z} /(g-1) \mathbb{Z}$ acts properly and freely on $\Sigma_{g}$, and the quotient space is $\Sigma_{2}$. Therefore $\pi_{1}\left(\Sigma_{g}\right)$ injects into $\pi_{1}\left(\Sigma_{2}\right)$ as a subgroup of index $(g-1)$ (in other words, $\Sigma_{g}$ is a $(g-1)$-sheeted regular covering of $\left.\Sigma_{2}\right)$. Consequently, $\pi_{1}\left(\Sigma_{g}\right)$ is in $\mathcal{A}$ by Theorem 20. Moreover, the fundamental group of a torus $\pi_{1}\left(\mathbb{T}^{2}\right)=\pi_{1}\left(\Sigma_{1}\right)$ is isomorphic to $\mathbb{Z}^{2}$, an amenable group. Therefore, we have:

Theorem 21. Let $\Sigma_{g}$ be a closed oriented surface of genus $g \geq 1$. The fundamental group $\Gamma_{g}=\pi_{1}\left(\Sigma_{g}\right)$ of $\Sigma_{g}$ admits a transitive, faithful and amenable action for all $g \geq 1$.

Corollary 22. For any compact surface $S$, the fundamental group $\pi_{1}(S)$ is in $\mathcal{A}$.

Proof. First of all, we can suppose that $S$ is oriented. In fact, it is well known that if $S$ is a non-oriented connected surface, then there exists a oriented 2-sheeted covering space $\widetilde{S}$ (cf. [6]). Then $\pi_{1}(\widetilde{S})$ is a subgroup of index 2 of $\pi_{1}(S)$ so that it is coamenable in $\pi_{1}(S)$ (a subgroup $H<G$ is co-amenable if the $G$-action on $G / H$ is amenable). Therefore, in order that $\pi_{1}(S) \in \mathcal{A}$, it suffices to have $\pi_{1}(\widetilde{S}) \in \mathcal{A}$ by Proposition 1 (vi) in [7].

If $S$ is a closed oriented surface (i.e., without boundary), $S$ is either a sphere or a finite connected sum of tori $\Sigma_{g}, g \geq 1$; so $\pi_{1}(S) \in \mathcal{A}$ in both cases. If $S$ is a surface with boundary components, then $\pi_{1}(S)$ is a free group (the fundamental group of a sphere with $p$ boundary components is a free group of rank $p-1$, and the fundamental group of $\Sigma_{g}$ with $p$ boundary components is a free group of rank $2 g+p-1$ for all $g \geq 1$ ), so it is again in $\mathcal{A}$ by van Douwen's theorem.

Example 5.1 (Surface bundles over $\mathbb{S}^{1}$ ). A surface bundle over $\mathbb{S}^{1}$ is a closed 3manifold which is constructed as a fiber bundle over the circle with fiber a closed surface. The fundamental group $G$ of such bundle can be viewed as an $\mathrm{HNN}$-extension

$$
\left.G=\pi_{1}\left(M_{\phi}\right)=\left\langle\Gamma_{g}, t\right| t x t^{-1}=\phi_{*}(x) \text { for all } x \in \Gamma_{g}\right\rangle,
$$

where $\phi: \Sigma_{g} \rightarrow \Sigma_{g}$ is a homeomorphism. Thus, we have a short exact sequence

$$
0 \rightarrow \Gamma_{g} \rightarrow G \rightarrow \mathbb{Z} \rightarrow 0 .
$$

The subgroup $\Gamma_{g}$ is co-amenable in $G$ since it is normal in $G$ and $G / \Gamma_{g} \simeq \mathbb{Z}$ is amenable. Therefore, we have $G \in \mathcal{A}$.

The Thurston's virtual fibration conjecture states that [16]: 
Every closed, irreducible, atoroidal 3-manifold $M$ has a finite-sheeted cover which fibres over the circle.

It follows from the conjecture that the fundamental group $\pi_{1}(M)$ is in $\mathcal{A}$ since it contains a finite index subgroup which is in $\mathcal{A}$.

\section{References}

[1] I. Agol, Criteria for virtual fibering. J. Topol. 1 (2008), 269-284. Zbl 1148.57023 MR 2399130311

[2] C. Anantharaman-Delaroche and J. Renault, Amenable groupoids. Monogr. Enseign. Math. 36, L'Enseignement Mathématique, Geneva 2000. Zbl 0960.43003 MR 1855241 309

[3] B. Bekka, P. de la Harpe and A. Valette, Kazhdan's property (T), New Math. Monogr. 11, Cambridge University Press, Cambridge 2008. Zbl 1146.22009 MR 2415834310

[4] G. Baumslag, On the residual finiteness of generalised free products of nilpotent groups. Trans. Am. Math. Soc. 106 (1963), 193-209. Zbl 0112.25904 MR 0144949329

[5] E. K. van Douwen, Measures invariant under actions of $\mathbb{F}_{2}$. Topology Appl. 34 (1990), 53-68. Zbl 0701.43001 MR 1035460309

[6] W. Fulton, Algebraic topology. Grad. Texts in Math. 153, Springer-Verlag, New York 1995. Zbl 0852.55001 MR 1343250330

[7] Y. Glasner and N. Monod, Amenable actions, free products and a fixed point property. Bull. Lond. Math. Soc. 39 (2007), 138-150. Zbl 05139551 MR 2303529 310, 329, 330

[8] F. P. Greenleaf, Invariant means on topological groups and their applications. Van Nostrand Math. Stud. 16, Van Nostrand Reinhold Co., New York 1969. Zbl 0174.19001 MR 0251549309

[9] R. Grigorchuk and V. Nekrashevych, Amenable actions of nonamenable groups. Zap. Nauchn. Sem. S.-Peterburg. Otdel. Mat. Inst. Steklov. (POMI) 326 (2005), 85-96; English transl. J. Math. Sci. 140 (2007), 391-397. Zbl 1127.43001 MR 2183217310

[10] N. Higson and J. Roe, Amenable group actions and the Novikov conjecture. J. Reine Angew. Math. 519 (2000), 143-153. Zbl 0964.55015 MR 1739727 309

[11] R. C. Lyndon and P. E. Schupp, Combinatorial group theory. Ergeb. Math. Grenzgeb. 89, Springer-Verlag, Berlin 1977. Zbl 0368.20023 MR 0577064328

[12] W. Magnus, A. Karrass, and D. Solitar, Combinatorial group theory. 2nd ed., Dover Publications Inc., Mineola, NY, 2004. Zbl 1130.20307 MR 2109550321

[13] J. von Neumann, Zur allgemeinen Theorie des Masses. Fund. Math. 13 (1929), 73-116. JFM 55.0151.01 JFM 55.0151.02 309

[14] N. Ozawa, Amenable actions and applications. In Proc. Internat. Congr. Mathematicians (Madrid, 2006). Vol. II, EMS Publ. House, Zürich 2006, 1563-1580. Zbl 1104.46032 MR 2275659309

[15] J-P. Serre, Arbres, amalgames, $S L_{2}$. Astérisque 46 (1977). Zbl 0369.20013 MR 0476875 314 
[16] W. P. Thurston, Three-dimensional manifolds, Kleinian groups and hyperbolic geometry. Bull. Amer. Math. Soc. (N.S.) 6 (1982), 357-381. Zbl 0496.57005 MR 648524330

[17] R. J. Zimmer, Amenable ergodic group actions and an application to Poisson boundaries of random walks. J. Funct. Anal. 27 (1978), 350-372. Zbl 0391.28011 MR 0473096309

Received October 13, 2008; revised March 2, 2009

S. Moon, Institut de Mathématiques, Université de Neuchâtel, 11, Rue Emile Argand - BP 158, 2009 Neuchâtel, Switzerland

E-mail: so.moon@unine.ch 